\title{
Factores socioeducativos y sentido de coherencia asociados a la calidad de vida en adolescentes de Lima Metropolitana
}

\author{
Mariela Dejo \\ María del Carmen Espinoza \\ Paola Gambini \\ Macarena Scarafia \\ Universidad de Lima, Perú
}

\begin{abstract}
Recibido: 21 de enero del 2018 / Aprobado: 26 de agosto del 2018 doi: 10.26439/persona2018.n021.3021
\end{abstract}

La investigación tuvo como objetivo explicar el constructo de calidad de vida asociado a factores socioeducativos y sentido de coherencia en adolescentes de Lima Metropolitana. Participaron 1211 adolescentes, entre 12 y 19 años de edad, de instituciones educativas públicas y privadas de Lima Metropolitana. Se administraron el Cuestionario de Evaluación de la Calidad de Vida de Alumnos Adolescentes (CCVA), la Escala de Sentido de Coherencia y un cuestionario sobre las variables de estudio: contexto familiar, clima escolar y factores de riesgo. Los resultados muestran que las tres dimensiones del sentido de coherencia son predictoras de la calidad de vida. Los factores socioeducativos familiar y clima escolar presentaron diferencias estadísticas significativas en los niveles de calidad de vida según sectores socioeconómicos.

calidad de vida / sentido de coherencia / adolescentes

Correo electrónico: mcdejo@ulima.edu.pe 


\section{Socio-educational factors and sense of coherence associated with quality of life in adolescents of Lima Metropolitan area}

The research aimed to explain the construct Quality of Life associated with socioeducational factors and the sense of coherence in adolescents of Lima Metropolitan area. The study population consisted of 1211 adolescents aged 12 to 19 attending public and private educational institutions of Lima Metropolitan area. Data was obtained through the Questionnaire for Assessing Adolescent Students' Quality of Life (CCVA), the Sense of Coherence Scale (SCS), and a questionnaire on the study variables Family Context, School Climate, and Risk Factors. The results show that the three dimensions of the sense of coherence are good predictors of quality of life. The socio-educational factors Family Context and School Climate presented statistically significant differences in quality of life levels depending on the socio-economic sectors. 


\section{INTRODUCCIÓN}

En los últimos años, ha surgido la necesidad de responder a una realidad social compleja y demandante: la educación escolar en el Perú. Para lograrlo, es imprescindible preparar a los estudiantes en logros académicos, pero también abordar competencias fundamentales para el desarrollo personal y la consolidación de un proyecto de vida. Por esta razón, el objetivo principal de las políticas públicas educativas actuales es brindar una formación integral al estudiante (Consejo Nacional de Educación, 2007). En este sentido, la psicología contribuye mediante investigaciones acerca de diversos factores asociados al éxito académico; sin embargo, la necesidad de conocer más al respecto para proponer pautas educativas que puedan ser implementadas en políticas públicas sigue vigente.

Actualmente, se conoce que los constructos de calidad de vida y sentido de coherencia se encuentran estrechamente relacionados con el bienestar y la satisfacción personal, ya que constituyen recursos adaptativos para afrontar las demandas y estresores de la vida cotidiana en todos los ámbitos de una persona: social, educativo, laboral y de la salud. No obstante, estos conceptos han sido poco explorados en la realidad peruana, principalmente en la población joven y en contextos educativos.

\section{Calidad de vida}

A la fecha, la propuesta más aceptada sobre la identificación de las principales dimensiones de la calidad de vida es la que proponen Schalock y Verdugo (2002): desarrollo personal, bienestar emocional, relaciones interpersonales, bienestar físico, bienestar material, autodeterminación, inclusión social y derechos. Si bien estas son similares para todas las personas, varían en intensidad y valoración; además, su evaluación depende de la cultura. Es así como, a partir de un exhaustivo análisis de estos componentes, se construyó el Cuestionario de Evaluación de la Calidad de Vida de Alumnos Adolescentes (CCVA) (Gómez-Vela y Verdugo, 2009), que evalúa aspectos relevantes de la vida y el grado de satisfacción con esta, el cual fue utilizado para realizar esta investigación.

\section{Sentido de coherencia}

Para Antonovsky y Sagy (1986), el sentido de coherencia es una capacidad y una orientación global que define la capacidad con la cual un individuo, con un persistente y dinámico sentimiento de confianza, encara los estímulos provenientes de los medios internos o externos. Dentro de este constructo, se pueden identificar tres subdimensiones: la capacidad de comprensión (medida en que la persona puede percibir los estímulos como estructurados, predictivos y explicables), la capacidad de gestión (percepción acerca de los propios recursos para satisfacer las exigencias colocadas por esos estímulos) y la capacidad de significado (percepción acerca del sentido de la vida y catalizador del esfuerzo que se invierte en ella). Cada una de las dimensiones del sentido de coherencia se ve influenciada por las experiencias vitales del individuo y sus recursos generales de resistencia (GRR) (Rivera, García-Moya, Moreno y Ramos, 
2013). Asimismo, Antonovsky (como se citó en Dejo, 2007) señala que el sentido de coherencia facilita el desarrollo de una estrategia de resolución de problemas y gestión de estrés, lo cual promueve la salud mental y la calidad de vida.

Del mismo modo, Antonovsky y Sagy (1986) resaltan el papel de la familia en la constitución de esta capacidad, indicando que la posición social, la situación económica y las relaciones sociales intervienen en la formación de este fenómeno. Los padres pueden resultar una pieza clave en la transformación positiva del potencial de los hijos y su apoyo se puede manifestar mediante diversas conductas, actividades y estrategias.

\section{Factores socioeducativos}

Existen diversos factores que influyen en la vida del adolescente. Por ejemplo, el rendimiento académico, que suele ser definido por los docentes a través de las calificaciones escolares, y usualmente se toma como sinónimo de inteligencia o de aprendizaje. Sin embargo, otros autores como Piñero y Rodríguez (1998) añaden otras múltiples variables, como el contexto del estudiante y su nivel socioeconómico. Así también, la autoconfianza, la autoestima, el clima escolar del aula y el maltrato a los estudiantes son factores vinculantes al rendimiento académico (Espinoza, 2006).

Asimismo, la familia es una pieza clave, es decir, una de las influencias educativas más poderosas para la transformación del potencial en talentos actuantes, no solo porque constituye el primer contexto de socialización del ser humano, sino también porque aporta "ingredientes" básicos a la educación de los niños con altas capacidades, como conocimientos, ambiente de libertad, seguridad, atención personalizada y educación permanente (De la Torre y Pérez, 2006). Esta influencia se da mediante diversas conductas, actividades y estrategias de los miembros, dentro y fuera de la escuela (Castellanos, Bazán, Ferrari y Hernández, 2015).

Otro factor que influye en el adolescente es el clima escolar, entendido como la percepción que los individuos tienen sobre los distintos aspectos del ambiente en el cual se desarrollan actividades educativas. El clima escolar puede ser de dos tipos: uno nutritivo, en el que la convivencia es positiva; y otro tóxico, donde se desarrolla el aspecto negativo de cada persona y la solución de conflictos es poco constructiva. Este factor impacta en áreas relevantes como la capacidad de retención de las escuelas, el bienestar y desarrollo socioafectivo de los alumnos, el bienestar de los docentes, el rendimiento y la efectividad escolar, entre otros (Milicic y Arón, 2000).

\section{Adolescencia}

Para evaluar todas las variables anteriormente mencionadas, es necesario tomar en cuenta que, durante la adolescencia, la persona atraviesa por un proceso de adaptación y búsqueda de identidad. Durante esta etapa, se adquieren los distintos estilos de vida que guiarán al individuo por el resto de su vida, tanto saludables como problemáticos (Díaz y González, 2014). Por tanto, este período es considerado como uno de los más importantes en la vida. En él se producen 
experiencias de gran impacto para el individuo, como el desarrollo de la identidad, la adquisición de distintas actitudes, la capacidad de razonamiento abstracto y el aumento de la autonomía (Organización Mundial de la Salud, OMS, 2016).

Sin embargo, actualmente, según el Ministerio de la Mujer y Poblaciones Vulnerables (2012), el adolescente en el Perú vive inmerso en una gran variedad de factores de riesgo que influyen en su desarrollo personal y su autorrealización. Ejemplos de ello son las crecientes tasas de maternidad adolescente, el consumo de drogas (legales e ilegales), las infecciones de transmisión sexual, los trastornos alimenticios, el trabajo adolescente, la explotación sexual, etcétera. Tales factores, en muchos casos, traen como consecuencia la deserción escolar.

\section{La educación en el Perú}

En cuanto a la educación en el Perú, el sistema nacional se caracteriza por presentar problemas de cobertura primaria $\mathrm{y}$ aún tiene mucho camino por recorrer, principalmente, en los niveles de inicial y secundaria. Cabe señalar que se observan amplias diferencias en cuanto a calidad educativa integral, según el nivel socioeconómico al que pertenece la familia del estudiante. En esta línea, el Proyecto Educativo Nacional al 2021 (Ministerio de Educación, 2007) menciona que una de las causas del fracaso es el centralismo educativo en el país, donde la expansión de la educación no ha cumplido su promesa de universalidad y calidad para todos. Además, se evidencia que el porcentaje del presupuesto público asignado a la educación es insuficiente. A esta situación la acompaña la falta de políticas que focalicen mayores recursos en las zonas con altos niveles de pobreza y necesidad.

La visión propuesta al 2021 aspira a que todos los niños desarrollen su potencial desde la primera infancia, accedan al mundo letrado, resuelvan problemas, practiquen valores, sepan seguir aprendiendo, se asuman ciudadanos con derechos $\mathrm{y}$ responsabilidades, entre otros. En este sentido, se han propuesto seis objetivos: (1) oportunidades y resultados de igual calidad para todos; (2) estudiantes e instituciones que logran aprendizajes pertinentes y de calidad; (3) maestros bien preparados que ejerzan la docencia con profesionalidad; (4) una gestión descentralizada, democrática, que alcanza resultados y es financiada con equidad; (5) educación superior de calidad que se convierta en un factor favorable para el desarrollo y la competitividad nacional; y (6) una sociedad que educa a sus ciudadanos y los compromete con su comunidad.

Una educación integral supone el desarrollo de la persona en todas y cada una de sus dimensiones: física, intelectual, social, moral y religiosa. Se plantea que, a lo largo de la vida, el individuo debe aprender a conocer, a hacer, a vivir y a ser; sin embargo, los sistemas educativos suelen dar prioridad y enfocarse únicamente en la adquisición de conocimientos, dejando de lado otras formas de aprendizaje. La educación debe ser vista como un todo, en el que el individuo sea capaz de desenvolverse en un amplio número de situaciones, comprender a otros y formar una personalidad 
propia, con autonomía, juicio y responsabilidad (Organización de las Naciones Unidas para la Educación, la Ciencia y la Cultura, Unesco, 1996).

Esta visión de educación puede resultar utópica para la realidad peruana, puesto que el sistema educativo nacional está centrado en potenciar la dimensión intelectual de sus alumnos, olvidando la importancia de las demás dimensiones. Sin embargo, incorporar en la educación escolar conceptos como calidad de vida y sentido de coherencia, desde una visión sistémica de aspectos salutogénicos y de bienestar personal vinculados a variables socioeducativas (como se plantea en esta investigación), podría ser de gran utilidad para lograr las transformaciones necesarias en el sistema educativo. Esto ayudaría a atender las principales necesidades y deseos de los estudiantes, de tal manera que se contribuya a su educación integral al contemplar todas las esferas de la vida de un individuo.

\section{Método}

\section{Tipo y diseño de investigación}

La presente investigación fue planteada desde un enfoque cuantitativo debido a la naturaleza de las variables, y es de tipo explicativo, ya que se pretende dar respuesta a las causas de los eventos sociales, en este caso, la influencia de los factores socioeducativos en los constructos de calidad de vida y sentido de coherencia en adolescentes de Lima Metropolitana. Con esta finalidad, el estudio siguió un diseño no experimental, debido a que no se buscó manipular de ninguna forma las variables antes mencionadas. Siendo así, el diseño fue transversal, pues se realizó una descripción y análisis de las relaciones causales que pudieron tener las variables en un momento determinado y en un contexto específico (Hernández, Fernández y Baptista, 2014).

\section{Participantes}

La población objetivo de la presente investigación estuvo constituida por adolescentes comprendidos entre las edades de 12 a 19 años que se encuentran cursando estudios en los centros educativos de Lima Metropolitana (Instituto Nacional de Estadística e Informática, INEI, 2014). En total, participaron 1211 estudiantes de instituciones educativas públicas y privadas, siendo el muestreo de tipo bietápico y habiéndose identificado a los participantes de los cinco ámbitos geográficos en que está distribuida Lima Metropolitana. La media de edad fue de 14 años y 9 meses; hubo un mayor porcentaje de mujeres $(54,6 \%)$ que de hombres $(45,4 \%)$. En lo que respecta al nivel socioeconómico, la mayoría de los adolescentes se encontraban en el nivel C (31,8\%), y en segundo orden estaba el nivel B (29,7 \%).

\section{Técnicas de recolección de datos}

A fin de lograr los objetivos de esta investigación, se emplearon tres instrumentos:

1. Cuestionario de Evaluación de la Calidad de Vida de Alumnos Adolescentes (CCVA), propuesto por Gómez-Vela y Verdugo (2009). El CCVA evalúa los siguientes aspectos: bienestar emocional, 
integración a la comunidad, relaciones interpersonales, desarrollo personal, bienestar físico, autodeterminación $\mathrm{y}$ bienestar material.

El cuestionario cuenta con estudios de validación en población peruana (Sotelo, Sotelo, Domínguez y Barboza, 2013); no obstante, se realizaron los análisis psicométricos respectivos para la población objeto de este estudio (evidencias de validez de contenido con el método de jueces, y evidencias de validez de la estructura interna a través del análisis factorial y confiabilidad con el alfa de Cronbach y las correlaciones ítem-test totales).

2. Escala de Sentido de Coherencia (Antonovsky, 1993). El test está constituido por 29 ítems agrupados en tres subescalas que evalúan las dimensiones del constructo: comprensibilidad, manejabilidad y significatividad. Los índices de fiabilidad reportados por Antonovsky (1993) oscilan entre .78 y .82. Eriksson y Lindström (2006) reportaron buenas evidencias de validez externa, mientras que Rivera, García-Moya, Moreno y Ramos (2013) reportan una confiabilidad de .87 en una muestra de adolescentes españoles.

3. Evaluación de los factores socioeducativos, para lo cual se elaboró un cuestionario donde se solicitó información sobre las variables de estudio: contexto familiar, clima escolar y riesgo de violencia. Se realizó un estudio piloto para obtener información psicométrica que permita garantizar el diseño y construcción del instrumento (evidencias de validez de contenido a través del método de jueces, y confiabilidad de las puntuaciones derivadas de la aplicación a la muestra piloto).

\section{Resultados}

Con respecto a los perfiles descriptivos de la calidad de vida de los adolescentes, y considerando el nivel socioeconómico al que pertenecen, las instituciones educativas del nivel socioeconómico $\mathrm{C}$ presentaron mayor respuesta promedio en las dimensiones de bienestar emocional $(3,19)$ e integración $(2,82)$. Asimismo, como se puede observar en la tabla 1, en el caso de la dimensión de las relaciones interpersonales, sobresalen las respuestas promedio en los colegios del nivel socioeconómico AB y B. Es interesante observar cómo en los colegios del nivel socioeconómico $\mathrm{AB}$ destacaron las respuestas promedio en las dimensiones de desarrollo personal $(3,35)$, autodeterminación $(3,16)$ y bienestar material $(3,03)$. Por último, en la dimensión de bienestar físico, las respuestas promedio se hallan en los colegios de sectores económicos $\mathrm{AB}, \mathrm{B}$ y $\mathrm{C}$ (3,43 en todos ellos). 
Tabla 1

Resumen descriptivo de la calidad de vida según nivel socioeconómico

\begin{tabular}{lccccccc}
\hline NSE & $\begin{array}{c}\text { Bienestar } \\
\text { emocional }\end{array}$ & Integración & $\begin{array}{c}\text { Relaciones } \\
\text { interpersonales }\end{array}$ & $\begin{array}{c}\text { Desarrollo } \\
\text { personal }\end{array}$ & $\begin{array}{c}\text { Bienestar } \\
\text { físico }\end{array}$ & $\begin{array}{c}\text { Auto- } \\
\text { determinación }\end{array}$ & $\begin{array}{c}\text { Bienestar } \\
\text { material }\end{array}$ \\
\hline AB & 3,06 & 2,77 & 3,37 & 3,35 & 3,43 & 3,16 & 3,03 \\
B & 3,11 & 2,78 & 3,37 & 3,24 & 3,43 & 3,12 & 2,97 \\
C & 3,19 & 2,82 & 3,33 & 3,29 & 3,43 & 3,06 & 2,89 \\
D & 3,13 & 2,74 & 3,26 & 3,25 & 3,39 & 3,01 & 2,84 \\
E & 3,18 & 2,70 & 3,31 & 3,23 & 3,33 & 3,01 & 2,98 \\
\hline K-S & & & & & & & \\
p & .09 & .16 & .01 & .12 & .71 & .02 & $<.0001$ \\
\hline
\end{tabular}

Sobre los perfiles descriptivos de las áreas de la Escala de Sentido de Coherencia, y teniendo en cuenta el nivel socioeconómico de los participantes, en la tabla 2 se encontró que en la dimensión de manejabilidad sobresale la respuesta promedio en los colegios del nivel socioeconómico $\mathrm{AB}$ $(4,74)$. En el caso de las dimensiones de significatividad y comprensibilidad, destaca la respuesta promedio en los colegios del nivel socioeconómico B (5,14 y 3,94, respectivamente).

Tabla 2

Resumen descriptivo del sentido de coherencia

\begin{tabular}{cccc}
\hline NSE & Manejabilidad & Significatividad & Comprensibilidad \\
\hline AB & 4,74 & 5,12 & 3,90 \\
B & 4,70 & 5,14 & 3,94 \\
C & 4,59 & 5,13 & 3,83 \\
D & 4,55 & 5,04 & 3,76 \\
E & 4,29 & 4,89 & 3,78 \\
\hline K-S & & & \\
P & .002 & .48 & .24 \\
\hline
\end{tabular}


En cuanto a los perfiles descriptivos por áreas de los factores socioeducativos según el nivel socioeconómico, en la tabla 3 se evidenció que en el factor familiar y escolar prevalecieron las respuestas promedio en los colegios del NSE C (3,10 y 3,04, respectivamente). En el caso del factor riesgo, existe mayor protección en los colegios del NSE B y C (1,92 y 1,90, también respectivamente).

Tabla 3

Resumen descriptivo de factores socioeducativos

\begin{tabular}{cccc}
\hline NSE & Familiar & Escolar & Riesgo \\
AB & 3,03 & 3,01 & 1,86 \\
B & 3,04 & 2,97 & 1,92 \\
C & 3,10 & 3,04 & 1,90 \\
D & 3,08 & 2,98 & 1,87 \\
E & 3,02 & 3,04 & 180 \\
K-S & & & $<.0001$ \\
P & .14 & .07 & \\
\hline
\end{tabular}

De acuerdo con el modelo explicativo que utiliza la técnica de regresión múltiple, las tres dimensiones del sentido de coherencia resultaron ser predictoras de la calidad de vida $(\mathrm{p}<.05)$. En la tabla 4 , se puede observar que el modelo es estadísticamente significativo $\left(\mathrm{F}_{(2,1198)}=25,74, \mathrm{p}<.0001\right), \mathrm{y}$ explica el $60 \%$ de la varianza total.

\section{Tabla 4}

Dimensiones del sentido de coherencia como predictoras de la calidad de vida

\begin{tabular}{lccc}
\hline \multicolumn{1}{c}{ Dimensiones } & $\begin{array}{c}\text { Coeficiente } \\
\text { estandarizado } \\
\text { (beta) }\end{array}$ & $\mathrm{t}$ & $\mathrm{p}$ \\
\hline (Constante) & 0,06 & 11,46 & $<.0001$ \\
Manejabilidad & 0,13 & 2,56 & .011 \\
Significatividad & 0,10 & 5,46 & $<.0001$ \\
Comprensibilidad & & 4,48 & .0001 \\
\hline \multicolumn{1}{c}{$r=.77$} & & $\mathrm{r}_{2}=60 \%$ \\
\hline
\end{tabular}


Los factores socioeducativos familia $y$ clima escolar también fueron predictores de esta misma variable $(\mathrm{p}<.05)$. Igualmente, en la tabla 5 se observan las diferencias en la calidad de vida de acuerdo con el NSE $(\mathrm{p}<.05)$. Además, se encuentra que el modelo es estadísticamente significativo $\left(\mathrm{F}_{(3,1198)}=50,27, \mathrm{p}<.0001\right)$, y permite explicar el $60 \%$ de la varianza total.

\section{Tabla 5}

Factores socioeducativos como predictores de la calidad de vida

\begin{tabular}{lccc}
\hline Factores & $\begin{array}{c}\text { Coeficiente } \\
\text { estandarizado } \\
\text { (beta) }\end{array}$ & $\mathrm{t}$ & $\mathrm{p}$ \\
\hline (Constante) & 0,40 & 17,05 & $<.0001$ \\
Familiar & 0,32 & 18,09 & $<.0001$ \\
Clima escolar & $-0,02$ & 14,74 & $<.0001$ \\
Riesgo & $-0,09$ & $-1,12$ & .263 \\
NSE & & $-4,95$ & $<.0001$ \\
\hline$r=.77$ & & $r_{2}=60 \%$ \\
\hline
\end{tabular}

Por otro lado, siguiendo con el mismo modelo, en la tabla 6 se demuestra que la dimesión de bienestar emocional es predictora del sentido de coherencia $(\mathrm{p}<.05)$.
Asimismo, se encuentra que el modelo es estadísticamente significativo $\left(\mathrm{F}_{(6,1198)}=\right.$ $65,74, \mathrm{p}<.0001)$, y permite explicar el $38 \%$ de la varianza total.

Tabla 6

Dimensiones de la calidad de vida como predictoras del sentido de coherencia

\begin{tabular}{lccc}
\hline & Coeficiente estandarizado (beta) & $\mathrm{t}$ & $\mathrm{p}$ \\
\hline (Constante) & & 1,39 & .146 \\
Bienestar emocional & 0,30 & 8,49 & $<.0001$ \\
Integración & $-0,01$ & $-0,23$ & .818 \\
Relaciones interpersonales & 0,05 & 1,36 & .177 \\
Desarrollo personal & $-0,04$ & $-1,09$ & .275 \\
Bienestar físico & 0,03 & 0,91 & .366 \\
Autodeterminación & 0,05 & 1,79 & .073 \\
Bienestar material & $-0,05$ & $-1,82$ & .070 \\
\hline$r=.62$ & & & $\mathrm{r}_{2}=38 \%$ \\
\hline
\end{tabular}


Por último, los factores socioeducativos familiar, escolar y riesgo también fueron predictores de esta misma variable $(p<.05)$. Se hallaron diferencias de acuerdo con el NSE de losparticipantes $(\mathrm{p}<.05)$. Elmodeloexplicativo alcanza el $38 \%$, según la tabla 7 . Además, se encuentra que el modelo es estadísticamente significativo $\left(\mathrm{F}_{(3,1198)}=47,51, \mathrm{p}<.0001\right)$, y permite explicar el $38 \%$ de la varianza total.

Tabla 7

Factores socioeducativos como predictores del sentido de coherencia

\begin{tabular}{lccc}
\hline & Coeficiente estandarizado (beta) & $\mathrm{t}$ & $\mathrm{p}$ \\
\hline (Constante) & & 1,45 & .149 \\
Familiar & 0,10 & 3,03 & .002 \\
Escolar & 0,13 & 3,98 & $<.001$ \\
Riesgo & 0,05 & 2,10 & .036 \\
NSE & $-0,07$ & $-2,85$ & .004 \\
\hline $\mathrm{r}=.62$ & & & $\mathrm{r}_{2}=38 \%$ \\
\hline
\end{tabular}

\section{DisCusión}

Los antecedentes de investigación en materia de calidad de vida adolescente muestran que la mayoría de estudios se han focalizado en países de elevados recursos y en personas con enfermedades crónicas de todas las especialidades médicas (HiguitaGutiérrez y Cardona-Arias, 2015). Dada la realidad local, resulta imperativo continuar investigando acerca de esta temática en poblaciones menos favorecidas económicamente y en adolescentes sin enfermedad.

El presente estudio revela que los adolescentes de nivel socioeconómico medio ofrecen mayor respuesta promedio en la dimensión de bienestar emocional, mientras que en niveles socioeconómicos más altos (AB) se evidencia mayor respuesta promedio en las dimensiones de relaciones interpersonales, autodeterminación y bienestar material. Por un lado, autores como Prieto et al. (2008) encontraron que el bienestar emocional es un factor determinante al valorar la calidad de vida. Por otro lado, hogares que satisfacen necesidades tanto en lo material como en lo social favorecen los sentimientos de satisfacción, bienestar general, seguridad, metas, creencias y espiritualidad de los jóvenes (Gómez-Vela y Verdugo, 2009). En ese sentido, el mayor acceso a recursos económicos puede brindar la oportunidad para que los jóvenes desarrollen capacitación en autorregulación, toma de decisiones y solución de problemas. Indicadores como elección personal, autonomía y satisfacción con el ámbito académico suelen presentarse en las personas que participan de entornos familiares, educativos y sociales más desarrollados. Además, las 
condiciones materiales con las que cuentan contribuyen a un mejor enfoque en sus proyectos personales y de autorrealización, e igualmente facilitan la interacción social, lo que genera en los adolescentes mayor integración a su comunidad, ajuste psicosocial, menor violencia y aislamiento, mayor autoestima académica y social, y más satisfacción con la vida (Martínez, Amador, Moreno y Musitu, 2011).

Con relación al sentido de coherencia, se encontró que mientras mayor es el nivel socioeconómico al que pertenecen los adolescentes, se presenta una percepción más segura de su entorno, sintiéndose en la capacidad de responder con éxito a situaciones demandantes de la vida (Salazar, Ruvalcaba, Figueroa y Pando, 2015). Esto se debe a que el poder adquisitivo hace posible el desarrollo de actividades familiares recreativas y que los padres puedan involucrarse en ellas, lo que promueve el sano desarrollo de relaciones parentales y de recursos de afrontamiento adecuados (Moreno, Muñoz-Tinoco y Pérez Moreno, citados por García-Moya, 2014). A contraparte, la disminución de la fuerza de este constructo se ve amenazada frecuentemente por dificultades económicas experimentadas durante la infancia (Lundberg, citado por García-Moya, 2014) y la posesión de pocos recursos materiales en la actualidad. García-Moya (2014) menciona que la falta de recursos genera consecuencias negativas para las relaciones entre padres e hijos. De acuerdo con los resultados obtenidos en la muestra estudiada de adolescentes en este trabajo, el sentido de coherencia es predictor de la calidad de vida, como también refieren Eriksson y Lindström
(2006), ya que cuanto mayor sea su sentido de coherencia, mejor será su calidad de vida. De forma inversa, los factores socioeducativos: familiar, escolar y riesgo, son predictores del sentido de coherencia de los participantes.

De las variables estudiadas, la que resulta de mayor poder explicativo sobre la calidad de vida de los participantes es el factor familiar. Diversos autores (De la Torre y Pérez, 2006; Freeman, 2010; Castellanos et al., 2015) han subrayado cómo la familia brinda apoyo a los niños desde sus primeras etapas en los planos afectivo, material e instrumental, dotándolos de estrategias y oportunidades para que potencien sus recursos con fines de adaptación. Del mismo modo, los factores sociofamiliares protectores de la calidad de vida son la funcionalidad familiar, la demostración de afecto entre los padres y el juego entre padres e hijos (Vilela-Estrada et al., 2016; Higuita-Gutiérrez y CardonaArias, 2014). Si es en la familia donde se forman los primeros vínculos de apego y se construyen conjuntamente las ideologías de la realidad, la presencia de conflicto entre los padres influye negativamente en la formación del sentido de coherencia en los hijos (Bowlby et al.; Schneider, Atkinson y Tardif, citados por García-Moya, 2014). Al absorber los problemas familiares, aumenta la irritabilidad y el agotamiento emocional, por lo que los individuos se vuelven más proclives al conflicto y se evidencia mayor presencia de prácticas punitivas (Buehler y Gerard, 2002). Por el contrario, factores protectores como las relaciones personales sólidas, así como un sistema personal de creencias y estrategias de afrontamiento 
positivas, fortalecen la prevención del suicidio (World Health Organization, WHO, 2014), mientras que un bajo sentido de coherencia se asocia a la ideación suicida alta, y viceversa (Ristkari, Sourander, Ronning, Nikolakaros y Helenius, 2008).

El clima escolar es el segundo predictor de la calidad de vida de los participantes. De ahí la necesidad de generar ambientes escolares nutritivos (Milicic y Arón, 2000), caracterizados por la no violencia, la convivencia e interacción social positivas, la buena disposición para los aprendizajes y la sensación de estar en agrado con las actividades desarrolladas. También es necesario que exista un compromiso y motivación de parte de las escuelas a través del desarrollo de relaciones positivas entre estudiantes, profesores y padres, lo que redundará en el incremento de la calidad de vida de los adolescentes (Arancibia, 1991; Bellei, Muñoz, Pérez y Raczynski, 2003).

Es de suma importancia señalar la deficiencia en el sistema educativo en instituciones del sector público, a diferencia de las del sector privado, en la realidad peruana. Así, se observa cómo en niveles socioeconómicos más bajos la educación impartida es rutinaria, pasiva y mecánica (Unicef, 2005). La motivación que los docentes puedan tener se obstaculiza por la presencia de condiciones laborales poco favorables para el profesorado, lo que repercute en los procesos de enseñanza-aprendizaje, el clima escolar, la satisfacción emocional y, por ende, en la calidad de vida de los adolescentes.

Para concluir, asociando las anteriores variables predictoras, se observa cómo el bienestar físico y psicológico, la funcionalidad familiar y el ambiente escolar influyen positivamente sobre la percepción de la calidad de vida. Si bien en el presente estudio no se ha encontrado relación significativa entre el factor riesgo y la calidad de vida, autores como HidalgoRasmussen e Hidalgo-San Martín (2015), y Margarita, Vinaccia, Zilena, y González (2014), evidenciaron que la disminución del ánimo, la desesperanza, el estrés y la depresión aumentan la posibilidad de un nivel de calidad de vida bajo, constituyendo comportamientos asociados a riesgo suicida, en contraposición a lo que factores protectores de la calidad de vida psicosocial y emocional podrían aportar.

\section{CONCLUSIONES}

Como resultado del estudio, se plantean las siguientes conclusiones:

1. Los estudiantes del nivel socioeconómico $\mathrm{C}$ presentaron mayores respuestas promedio en las dimensiones de bienestar emocional e integración de la calidad de vida, mientras que en los niveles socioeconómicos $\mathrm{AB}$ sobresalen respuestas promedio en desarrollo personal, autodeterminación y bienestar material. En estas dos últimas dimensiones, se encuentran, además, diferencias estadísticamente significativas entre niveles socioeconómicos.

2. La dimensión de manejabilidad del sentido de coherencia tiene mayores respuestas promedio en el nivel socioeconómico $\mathrm{AB}$. Además, es en esta dimensión donde se encuentran diferencias es- 
tadísticamente significativas en función de los niveles socioeconómicos.

3. En el nivel C, sobresalen las respuestas promedio en los factores socioeducativos familiar y clima escolar. En el factor riesgo, hay mayor protección en los colegios de los sectores B y C. Este factor tiene diferencias significativas en función del nivel socioeconómico.

4. Las tres dimensiones del sentido de coherencia son predictoras de la calidad de vida. El modelo es explicado en un $60 \%$.

5. Los factores socioeducativos familiar y clima escolar son predictores de la calidad de vida en adolescentes, hallándose diferencias significativas en los niveles de calidad de vida según sectores socioeconómicos.

6. La dimensión de bienestar emocional de la calidad de vida es predictora del sentido de coherencia.

7. Los factores socioeducativos familiar, escolar y riesgo son predictores del sentido de coherencia. El modelo es explicado en un $38 \%$. También hay diferencias en el sentido de coherencia en función del nivel socioeducativo perteneciente.

8. Las instituciones educativas deben prestar atención a la mejora de las relaciones sociales (amigos, docentes, familiares), y no solo a aspectos de enseñanza y aprendizaje, para lograr un buen ajuste personal.

9. Se destaca la importancia trascendental de la familia en los procesos de desarrollo personal, sobre todo en aspectos de bienestar, calidad de vida y satisfacción personal.

10. Se debe priorizar la educación de la niñez y adolescencia, y, específicamente en el currículo escolar, los aspectos de desarrollo personal que involucren autogestión de recursos adaptativos en el ámbito psicosocial, toma de decisiones, autopercepción y significado de la propia vida, autonomía, autoeficacia, entre otros componentes de la salud emocional, que permitan darle una visión integral al desarrollo del niño y del adolescente.

\section{RefERENCIAS}

Antonovsky, A. (1993). The Structure and Properties of the Sense of Coherence Scale. Social Science \& Medicine, 36, 725-736.

Antonovsky, H., y Sagy, S. (1986). The Development of a Sense of Coherence and Its Impact on Responses to Stress Situations. Journal of Social Psychology, 126(2), 213-225.

Arancibia, V. (1991). Efectividad escolar: un análisis comparado. Recuperado de www. cepchile.cl/dms/archivo_1819_1298/rev47_arancibia.pdf 
Bellei, C., Muñoz, G., Pérez, L. M., y Raczynski, D. (2003). ¿Quién dijo que no se puede? Escuelas efectivas en sectores de pobreza. Santiago de Chile: Ministerio de Educación; Unicef.

Buehler, Ch., y Gerard, J. M. (2004). Marital Conflict, Ineffective Parenting, and Children's and Adolescent' Maladjustment. Journal of Marriage and Family, 64, 78-92

Castellanos, D., Bazán, A., Ferrari, A., y Hernández, C. (2015). Apoyo familiar en escolares de alta capacidad intelectual de diferentes contextos socioeducativos. Revista de Psicología, 33(2), 299-332.

Consejo Nacional de Educación. (2007). Proyecto Educativo Nacional. Recuperado de http:// www.cne.gob.pe/

De la Torre, F., y Pérez, L. (2006). La familia y el desarrollo del potencial creativo de los niños con altas capacidades. En L. Pérez (Coord.), Alumnos con capacidad superior: experiencias de intervención educativa (pp. 353-392). Madrid: Síntesis.

Dejo, M. (2007). Sentido de coherencia, afrontamiento y sobrecarga en cuidadores familiares de ancianos con enfermedad crónica. Avances en Psicología, 25(1), 64-71. Recuperado de http://www.scielo.org.co/pdf/apl/v25n1/v25n1a7.pdf

Díaz, C., y González, M. (2014). Conductas problema en adolescentes en la ciudad de Monterrey, México. Enfermería Global, 13(33), 1-16. doi: 10.6018/eglobal.13.1.184861

Eriksson, M., y Lindström, B. (2006). Antonovsky's Sense of Coherence Scale and the Relation with Quality of Life: A Systematic Review. Journal of Epidemiology and Community Health, 61, 938-944. doi: 10.1136/jech.2006.056028

Espinoza, E. (2006). Impacto del maltrato escolar en el rendimiento académico. Electronic Journal of Research in Educational Psychology, 4(9), 221-238.

Fondo de las Naciones Unidas para la Infancia (Unicef). (2005). La niñez en las políticas de educación: situación y propuestas. Recuperado de http://www.unicef.org/peru/ spanish/peru_politicas_educacion.pdf

Freeman, J. (2010). Families, the Essential Context for Gifts and Talents. En K. A. Heller, F. J. Monks, R. Sternberg y R. Subotnik (Eds.), International Handbook of Research and Development of Giftedness and Talent (pp. 573-585). Oxford: Pergamon Press.

García-Moya, I. (2014). El sentido de coherencia en la adolescencia. La contribución de la familia y otros contextos a su desarrollo y repercusión sobre la salud (tesis doctoral). Universidad de Sevilla, España. Recuperado de http://fondosdigitales. us.es/tesis/tesis/2288/el-sentido-de-coherencia-en-la-adolescencia-la-contribucionde-la-familia-y-otros-contextos-su-desarrollo-y-su-repercusion-sobre-la-salud/

Gómez-Vela, M., y Verdugo, M. A. (2009). Cuestionario de Evaluación de la Calidad de Vida en Alumnos Adolescentes (CCVA). Madrid: CEPE. 
Hernández, R., Fernández-Collado, C., y Baptista, P. (2014). Metodología de la investigación. México, D. F.: McGraw-Hill Interamérica.

Hidalgo-Rasmussen, C., e Hidalgo-San Martín, A. (2015). Comportamientos de riesgo de suicidio y calidad de vida, por género, en adolescentes mexicanos, estudiantes de preparatoria. Ciência \& Saúde Coletiva, 20(11), 3437-3445.

Higuita-Gutiérrez, L., y Cardona-Arias, J. (2014). Calidad de vida de adolescentes escolarizados de Medellín-Colombia. Revista Facultad Nacional de Salud Pública, 34(2), 145-155.

Higuita-Gutiérrez, L., y Cardona-Arias, J. (2015). Calidad de vida relacionada con la salud en adolescentes: revisión sistemática de las investigaciones publicadas en el período 1970-2013. Revista Médicas UIS, 28(1), 23-30.

Instituto Nacional de Estadística e Informática (INEI). (2014). Una mirada a Lima Metropolitana. Recuperado de https://www.inei.gob.pe/media/MenuRecursivo/ publicaciones_digitales/Est/Lib1168/libro.pdf

Margarita, J., Vinaccia, S., Zilena, L., y González, D. (2014). Influencia de los factores salutogénicos y patogénicos sobre la calidad de vida de adolescentes colombianos. Revista Argentina de Clínica Psicológica, 23(1), 81-93.

Martínez, B., Amador, L., Moreno, D., y Musitu, G. (2011). Implicación y participación comunitarias y ajuste psicosocial en adolescentes. Psicología y Salud, 21, 205-214.

Milicic, N., y Arón, A. (2000). Climas sociales tóxicos y climas sociales nutritivos para el desarrollo personal en el contexto escolar. Psykhe, 9(2), 117-123.

Ministerio de la Mujer y Poblaciones Vulnerables. (2012). Plan Nacional de Acción por la Infancia y Adolescencia 2012-2021. Recuperado de http://www.unicef.org/peru/ spanish/PNAIA-2012-2021.pdf

Ministerio de Educación. (2007). Proyecto Educativo Nacional al 2021. La educación que queremos para el Perú. Recuperado de http://www.minedu.gob.pe/DeInteres/xtras/ PEN-2021.pdf

Organización de las Naciones Unidas para la Educación, la Ciencia y la Cultura (UNESCO). (1996). La educación encierra un tesoro. Recuperado de http://unesdoc.unesco.org/ images/0010/001095/109590so.pdf

Organización Mundial de la Salud (OMS). (2016). Desarrollo en la adolescencia. Recuperado de http://www.who.int/maternal_child_adolescent/topics/adolescence/dev/es/

Piñero, L. J., y Rodríguez A. (1998). Los insumos escolares en la educación secundaria y su efecto sobre el rendimiento académico de los estudiantes: un estudio en Colombia (Spanish) [LCSHD Paper Series n. ${ }^{\circ}$ 36]. Washington D. C.: World Bank Group.

Prieto, M., Fernández, G., Rojo, F., Lardiés, R., Rodríguez, V., Ahmed, K., y Rojo, J. (2008). Factores sociodemográficos y de salud en el bienestar emocional como dominio de 
calidad de vida de las personas mayores en la comunidad de Madrid, 2005. Revista Española de Salud Pública, 82(3), 301-313. Recuperado de http://scielo.isciii.es/ scielo.php?script $=$ sci_arttext\&pid=S1135-57272008000300006

Ristkari, T., Sourander, A., Ronning, J. A., Nikolakaros, G., y Helenius, H. (2008). Life Events, Self-Reported Psychopathology and Sense of Coherence Among Young Men. A Population-Based Study. Nordic Journal of Psychiatry, 62, 464-467. doi: 10. 1080/08039480801984313

Rivera, F., García-Moya, I., Moreno, C., y Ramos, P. (2013). Developmental Contexts and Sense of Coherence in Adolescence: A Systematic Review. Journal of Health Psychology, 18, 800-812. doi: 10.1177/1359105312455077

Salazar, J., Ruvalcaba, N., Figueroa, N., y Pando, M. (2015). Sentido de coherencia y calidad de vida en trabajadores que participan en un programa de bienestar. Revista Iberoamericana de Psicología: Ciencia y Tecnología, 8(2), 91-98. Recuperado de https://dialnet.unirioja.es/ejemplar/435825

Schalock, R., y Verdugo, M. (2002). Handbook of Quality of Life for Human Services Practitioners. Washington, D. C.: AAMR.

Sotelo, N., Sotelo, L., Domínguez, S., y Barboza, M. (2013). Estudio corporativo de la calidad de vida de niños y adolescentes escolarizados de Lima y Áncash. Avances en Psicología, 21(1), 65-71.

Vilela-Estrada, M., Solano, F., Meza-Liviapoma, J., Araujo-Chumacero, M., Vilela-Estrada, A., y Mejía, C. (2016). Factores sociofamiliares asociados a la calidad de vida en niños de colegios de la ciudad de Piura, Perú. Revista Chilena de Pediatría. Recuperado de http://ebscohost.com

World Health Organization (WHO). (2014). Preventing Suicide: A Global Imperative. Recuperado de http://apps.who.int/iris/bitstream/10665/136083/1/9789275318508 spa.pdf 
